\title{
DEAD-box helicase 27 plays a tumor-promoter role by regulating the stem cell-like activity of human colorectal cancer cells
}

\author{
Chunxing Yangl,* \\ Daojiang $\mathrm{Li}^{1}$,* \\ Yang Bai' \\ Shenglei Song' \\ Peicheng Yan' \\ Runliu Wu' \\ Yi Zhang' \\ Gui Hu' \\ Changwei Lin' \\ Xiaorong $\mathrm{Li}^{\prime}$ \\ Lihuang Huang ${ }^{2}$
}

'Department of Gastrointestinal Surgery, The Third Xiangya Hospital of Central South University, Changsha, Hunan Province, China; ${ }^{2}$ Department of Central laboratory, The Third Xiangya Hospital of Central South University, Changsha, Hunan Province, China

*These authors contributed equally to this work
This article was published in the following Dove Medical Press journal:

OncoTargets and Therapy

\begin{abstract}
Background: Cancer stem cells (CSCs) are responsible for all important characteristics of tumors. DEAD-box helicase 27 (DDX27) is a member of the DEAD-box RNA helicase family, and there have been only a few studies on DDX27 function in cancer cells. This study is aimed at exploring whether DDX27 has any relation to tumorigenesis of colorectal cancer (CRC) and elucidating the potential mechanism.
\end{abstract}

Methods: Data from Catalog Of Somatic Mutations In Cancer, Gene Expression Omnibus, and The Cancer Genome Atlas databases reveal that DDX27 is overexpressed in CRC tissues. qRT-PCR and Western blots were used to evaluate the expression level of DDX27 in 40 paired clinical CRC samples. DDX27 was knockdown in HT29 and HCT116 cell line with shRNA. Then CCK-8, colony formation assay and flow cytometry assay were performed to examine proliferative ability, cell cycle and sensitivity to 5-fluorouracil. Sphere-formation assay and in vivo subcutaneous tumor-formation assay were used to assess self-renewal in vitro and vivo as well as the tumor-initiating potential.

Results: DDX27 is upregulated in CRC tissues and downregulation of DDX27 inhibits proliferation of colorectal cancer cell and promotes sensitivity to 5-fluorouracil. Downregulation of DDX27 can downregulate the gene expression of known CSC markers in CRC cells, inhibit sphere-formation ability, and promote colonosphere differentiation. Downregulation of DDX27 in CSCs can decrease the tumor-initiating ability of CRC cells in vivo.

Conclusion: DDX27 may play a tumorpromoter role of CRC by regulating the stem cell-like activity of CRC cells.

Keywords: DDX27, cancer stem cell, colonosphere, tumor-initiating ability

\section{Introduction}

Colorectal cancer (CRC) is one of the most commonly diagnosed cancers; it is ranked third in males and second in females worldwide. ${ }^{1} \mathrm{CRC}$ is one of the most deadly cancers, and, based on the data from 2000 to 2011, its incidence rate is increasing in China. ${ }^{2}$ Moreover, the development of CRC is a multistep process and CRC cells display intraclonal heterogeneity. ${ }^{3}$ The average survival time of advanced CRC patients has been obviously extended because of the improvement in therapeutic methods over the past two decades, but, as determined in the USA, most of these patients usually die within 3 years. ${ }^{4}$ Therefore, finding more effective therapy targets is urgently needed.

Cancer stem cells (CSCs) are defined as a subset of tumor cells that possess the capacity to self-renew and give rise to heterogeneous lineages of cancer cells that comprise the tumor. The alternative names are "tumor-initiating cells" and "tumorigenic cells". ${ }^{5}$ CSCs, as a stem cell-like population, can be identified by markers such
Correspondence: Changwei Lin Department of Gastrointestinal Surgery, The Third Xiangya Hospital of Central South University, Tongzi Po Road, No 172, Changsha, Hunan Province, China

Tel/fax +86 73I $886 \mid 8832$

Email linchangweil987@csu.edu.cn 
as CD44, CD133, ALDH, and so on. ${ }^{6}$ Like normal stem cells, CSCs can self-renew and preserve an undifferentiated stem cell population that can generate progenies at various stages of differentiation. ${ }^{7}$ Increasing evidence suggests that CSCs are responsible for all of the important characteristics of tumors, such as tumor initiation, ${ }^{8}$ proliferative ability, ${ }^{9}$ metastasis, ${ }^{10}$ therapy resistance, ${ }^{11}$ and recurrence. ${ }^{12}$

The DEAD-box RNA helicase family belongs to the helicase superfamily $2,{ }^{13}$ and its highly conserved motif II, which contains the Asp(D)-Glu(E)-Ala(A)-Asp(D) sequence, gave the DEAD-box name to this family. ${ }^{14}$ Members of this family are involved in various processes associated with RNA metabolism both in the nucleus and the cytoplasm. ${ }^{15}$ Mutations and deregulation of these family members have been linked to cancers. Increased expression of DDX21 was associated with increased c-Jun activity and rRNA processing in breast cancer, which drives breast tumorigenesis. ${ }^{16}$ The upregulation of DDX31 in renal cell carcinomas plays an oncogenic role through the regulation of the p53-HDM2 pathway. ${ }^{17}$ Importantly, Tsukamoto et al reported that DDX27 contributed to the colony-forming ability of gastric cancer cells and correlated it with poor prognosis in gastric cancer. ${ }^{18}$

In the present study, we investigated the effects of DDX27 on the stem cell-like features of CRC cells. We found that in CRC, DDX27 plays a tumor-promoter role by regulating the stem cell-like activity of CRC cells.

\section{Materials and methods}

\section{Human tissue samples and cell lines}

Human tissue samples of CRC and matched non-tumor tissues (ie, at least $5 \mathrm{~cm}$ away from the tumor edge) were obtained from patients who had undergone surgical treatment at the Third Xiangya Hospital of Central South University. All examined tumor tissues were sporadic adenocarcinomas. The use of tissues for this study was approved by the ethics committee of the Third Xiangya Hospital of Central South University, and the study was conducted in accordance with the Declaration of Helsinki. Before using these clinical materials for research purposes, all patients signed an informed consent. None of these patients received any preoperative chemotherapy or radiotherapy. The human CRC cell lines (HCT116 and HT29) were purchased from commercial sources and cultured in the Central Laboratory of the Third Xiangya Hospital of Central South University. HCT116 and HT29 cells were cultured in McCoy's 5A medium (KeyGEN BioTECH, Nanjing, China) and supplemented with 10\% FBS (BioInd, Kibbutz Beit Haemek, Israel) in a humidified atmosphere with $5 \% \mathrm{CO}_{2}$ at $37^{\circ} \mathrm{C}$.

\section{shRNA knockdown experiments}

The shRNA lentiviral vectors used for knockdown of DDX27 were synthesized by Genepharma Company (Suzhou, China). The following shRNA sequences were used for the DDX27 knockdown experiment: shRNA1 (5'-GCTTGCGGA CCTCGGCTTAAT-3'), shRNA2 (5'-GCTGATTTCAAC CCTGATTTC-3'), shRNA3 (5-GGAGAAAGAAGCAAA GGAAGG-3'), and the negative control (5'-TTCTCCGAAC GTGTCACGT-3'). We transfected cell lines with lentiviruses carrying these shRNA plasmids according to the manufacturer's instructions. shRNA3 was chosen as the representative shRNA because it was the most efficient one. The evidence in the protein level change of knockdown of DDX27 by shRNA3 sequences in HCT116 and HT29 lines is provided in Figure S1.

\section{RNA extraction and real-time PCR}

For real-time PCR analyses, total RNA was extracted by Trizol (Thermo Fisher Scientific, Waltham, MA, USA) and cDNA was synthesized using ReverTra Ace qPCR RT Master Mix with gDNA Remover (TOYOBO), following the manufacturer's recommendations. Quantitative PCR (qPCR) assays were performed by using KOD SYBR ${ }^{\circledR}$ qPCR Mix (TOYOBO) on LightCycler ${ }^{\circledR}$ 480II System (Hoffman-La Roche Ltd), according to the manufacturer's instructions. The primers used in this study can be found in Table 1 .

\section{Western blots}

Total proteins were extracted by lysis in radioimmunoprecipitation assay buffer (Nanjing KeyGen Biotech, Nanjing, China) and protease inhibitor cocktail (Nanjing KeyGen Biotech) for 15 minutes at $4^{\circ} \mathrm{C}$ and centrifuged at $12,000 \mathrm{rpm}$

Table I Primers used in this research

\begin{tabular}{|c|c|c|}
\hline $\begin{array}{l}\text { Gene } \\
\text { detected }\end{array}$ & Primer & Sequence $\left(5^{\prime}-3^{\prime}\right)$ \\
\hline CD44 & $\begin{array}{l}\text { Forward } \\
\text { Reverse }\end{array}$ & $\begin{array}{l}\text { CTGCCGCTTTGCAGGTGTA } \\
\text { CATTGTGGGCAAGGTGCTATT }\end{array}$ \\
\hline CDI33 & $\begin{array}{l}\text { Forward } \\
\text { Reverse }\end{array}$ & $\begin{array}{l}\text { GGCCCAGTACAACACTACCAA } \\
\text { ATTCCGCCTCCTAGCACTGAA }\end{array}$ \\
\hline EрCAM & $\begin{array}{l}\text { Forward } \\
\text { Reverse }\end{array}$ & $\begin{array}{l}\text { ATAACCTGCTCTGAGCGAGTG } \\
\text { TGCAGTCCGCAAACTTTTACTA }\end{array}$ \\
\hline LGR5 & $\begin{array}{l}\text { Forward } \\
\text { Reverse }\end{array}$ & $\begin{array}{l}\text { CACCTCCTACCTAGACCTCAGT } \\
\text { CGCAAGACGTAACTCCTCCAG }\end{array}$ \\
\hline TFF3 & $\begin{array}{l}\text { Forward } \\
\text { Reverse }\end{array}$ & $\begin{array}{l}\text { CTCCAGCTCTGCTGAGGAGT } \\
\text { GCTTGAAACACCAAGGCACT }\end{array}$ \\
\hline MUC2 & $\begin{array}{l}\text { Forward } \\
\text { Reverse }\end{array}$ & $\begin{array}{l}\text { ACCCGCACTATGTCACCTTC } \\
\text { GGACAGGACACCTTGTCGTT }\end{array}$ \\
\hline $\mathrm{DD} \times 27$ & $\begin{array}{l}\text { Forward } \\
\text { Reverse }\end{array}$ & $\begin{array}{l}\text { AGCCCGTGGACTTGACATTG } \\
\text { GCATCTTCCGCTCATCTTCTC }\end{array}$ \\
\hline GAPDH & $\begin{array}{l}\text { Forward } \\
\text { Reverse }\end{array}$ & $\begin{array}{l}\text { ACCTGACCTGCCGTCTAGAA } \\
\text { TCCACCACCCTGTTGCTGTA }\end{array}$ \\
\hline
\end{tabular}


for 15 minutes at $4^{\circ} \mathrm{C}$. The protein concentrations were measured by bicinchoninic acid protein assay. Proteins $(40 \mu \mathrm{g})$ were separated by $10 \%$ SDS-PAGE and transferred to polyvinylidene difluoride membranes. Membranes were blocked with 5\% non-fat milk in PBS for 2 hours at room temperature and incubated with primary antibodies at $4{ }^{\circ} \mathrm{C}$ overnight. Membranes were incubated with HRP-conjugated goat anti-rabbit IgG (1:15,000; LI-COR, Lincoln, NE, USA) for 2 hours at room temperature. The primary antibodies used were anti-DDX27 (Proteintech) and anti-GAPDH (Proteintech) at a 1:1,000 dilution.

\section{Cell proliferation assay}

Aliquots $(100 \mu \mathrm{L})$ containing 5,000 HCT116 cells or 2,500 HT29 cells were placed into the experimental wells of 96-well plates and cultured overnight. Ten microliters of Cell Counting Kit-8 (CCK-8; Dojindo, Kumamoto, Japan) were added into each well at timepoints of 24, 48, 72, and 96 hours after cell seeding. Cells were incubated with CCK-8 for 3 hours, and then the absorbance was measured at $450 \mathrm{~nm}$ with the BIOTEK ELX800 instrument (BIOTEK Instruments Inc., Winooski, VT, USA).

\section{Colony-formation assay}

A total of 500 cells were cultured in each well of a six-well plate for 9 days. Cell colonies were fixed with methyl alcohol for 30 minutes and then stained with $0.1 \%$ crystal violet for 30 minutes and photographed, after which the colonies were counted.

\section{Cell cycle assays}

For the cell cycle assay, cells were trypsinized and fixed with $70 \%$ ethanol at $4^{\circ} \mathrm{C}$ overnight. Then, $2 \times 10^{5}$ cells were resuspended in $0.5 \mathrm{~mL}$ propidium iodide/RNase Staining Buffer (BD Biosciences, San Jose, CA, USA). After 15 minutes of incubation at room temperature and protection from light, the cells were characterized. Data were acquired using a BD FACS Verse apparatus and analyzed using the Modfit software. The experiments were carried out in triplicate.

\section{Drug sensitivity assay}

Cells were cultured in complete medium containing defined concentrations ( $5 \mu \mathrm{g} / \mathrm{mL}$ for HCT116, $30 \mu \mathrm{g} / \mathrm{mL}$ for HT29) of 5-fluorouracil (5-FU; KeyGEN BioTECH) for 3 days. Then, the cells were trypsinised with EDTA-free trypsin and resuspended in $1 \times$ binding buffer (BD Biosciences) at $2.5 \times 10^{6}$ cells $/ \mathrm{mL}$ together with the cells floating in the medium. An aliquot $(100 \mu \mathrm{L})$ of this cell suspension was incubated with $5 \mu \mathrm{L}$ of allophycocyanin (APC)-Annexin V (BD Biosciences) and $5 \mu \mathrm{L}$ of 7-aminoactinomycin D (BD Biosciences) for 15 minutes in the dark. The reaction was terminated with the addition of $400 \mu \mathrm{L}$ of $1 \times$ binding buffer, and data were acquired using a BD FACS Verse apparatus and analyzed using the FlowJo software. For each experiment, $1 \times 10^{4}$ cells were analyzed. APC-Annexin V-positive cells were considered apoptotic, and the experiments were carried out in triplicate.

\section{Sphere-formation assay}

For the sphere-formation assay, cells were cultured in stem cell medium (SCM) containing DMEM/F-12 (Thermo Fisher Scientific), B-27 supplement (1:50; Thermo Fisher Scientific), $20 \mathrm{ng} / \mathrm{mL}$ human epidermal growth factor (EGF; PeproTech, Inc., Rocky Hill, NJ, USA), 10 ng/mL human fibroblast growth factor $\beta$ (FGF- $\beta$ ) (PeproTech, Inc.), $40 \mu \mathrm{g} / \mathrm{mL}$ heparin sodium salt (NeoFROXX GmbH, Einhausen, Germany), and penicillin/streptomycin (1:100; KeyGEN BioTECH). The principal components of this SCM were derived from Refs 1 and $\underline{2}$. Fixed amounts, 500 and 1,000 cells, were cultured in SCM in six-well plates in triplicate for 9 days in order to calculate the sphere numbers and sizes to evaluate the sphere-formation ability, and then, normal medium containing $2 \%$ FBS was used to induce differentiation. Twenty thousand cells were cultured in SCM in six-well plate in sextuplicate for 6 days for an in vivo subcutaneous tumor-formation assay.

\section{In vivo subcutaneous tumor-formation assay}

Twenty male Balb/c nude mice, 4-6 weeks of age and with body weight ranging between 18 and $22 \mathrm{~g}$, were obtained from the Department of Laboratory Animals of Central South University. These mice were randomly divided into four groups as follows: 1) HT29 shNC parental, 2) HT29 shDDX27 parental, 3) HT29 shNC colonospheres, and 4) HT29 shDDX27 colonospheres, with five mice in each group. Cells $\left(1 \times 10^{5}\right)$ in $100 \mu \mathrm{L}$ PBS were inoculated subcutaneously in the right flank of each group. Tumor nodules were measured every 2 days after their lengths exceeded $2 \mathrm{~mm}$, and the tumor volumes $(V)$ were calculated using the following formula: $V=\left(\right.$ width $^{2} \times$ length $) / 2$. Tumors were collected and photographed at 6 weeks. All animal experimental procedures used in this study were approved by the Animal Ethics Committee of Central South University and conducted in accordance with the Guideline of the Care and Use of Laboratory Animals in Central South University. $\mathrm{H} \& \mathrm{E}$ staining was performed on the xenograft tumors excised 
from nude mice, and the representative pictures are provided in Figure S2.

\section{Statistical analysis}

Data are expressed as the mean $\pm \mathrm{SD}$. Student's $t$-test was used for comparisons between groups, and $P<0.05$ was considered as a statistically significant difference.

\section{Results}

\section{DDX27 is upregulated in CRC tissues}

The information obtained from the Catalog Of Somatic Mutations In Cancer (COSMIC) database showed that DDX27 ranked fourth among the top ten genes, which were overexpressed in CRC samples, and large intestine cancer tissues had the highest overexpression percentages in various cancer samples (Figure 1A and B). Then, analysis of the data from The Cancer Genome Atlas (TCGA) and Gene Expression Omnibus databases showed that DDX27 was upregulated in CRC tissues compared with a normal control (Figure 1C and D). Consistent with the data retrieved from databases, analysis of the clinical CRC samples showed higher protein and mRNA expression levels of DDX27 (Figure 1E and F).

\section{Downregulation of DDX27 inhibits CRC cell proliferation, retards GI/S transition, and promotes sensitivity to 5 -FU}

As the expression level of DDX27 was significantly increased in CRC, this suggested that DDX27 might play a promoter role in CRC. Therefore, we tested if DDX27 knockdown repressed the proliferation of HCT116 and HT29 cells,
A

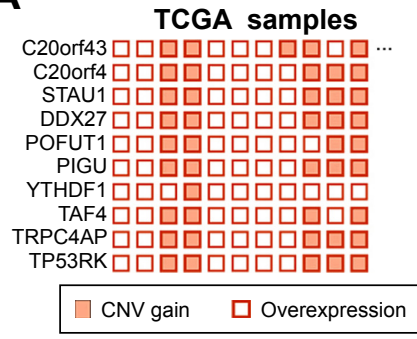

B

\begin{tabular}{lll}
\hline Tissue & \multicolumn{2}{c}{ DDX27 expression } \\
\cline { 2 - 3 } & Overexpression (\%) & Overexpression/tested \\
\hline Large intestine & 59.84 & $365 / 610$ \\
Stomach & 41.4 & $118 / 285$ \\
Ovary & 27.07 & $72 / 266$ \\
Urinary tract & 26.72 & $109 / 408$ \\
Esophagus & 25.6 & $32 / 125$ \\
\hline
\end{tabular}

C

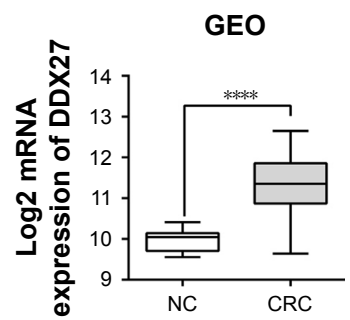

D

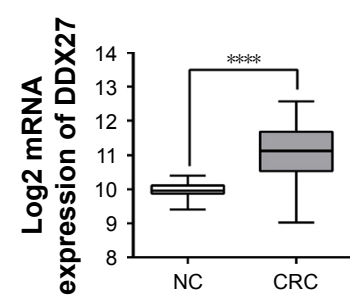

E

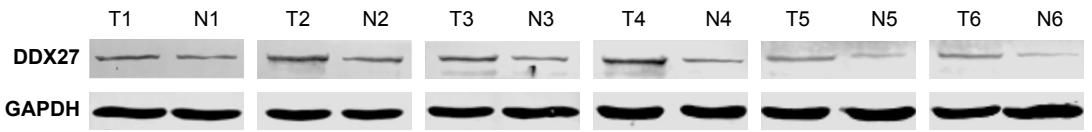

$\mathbf{F}$

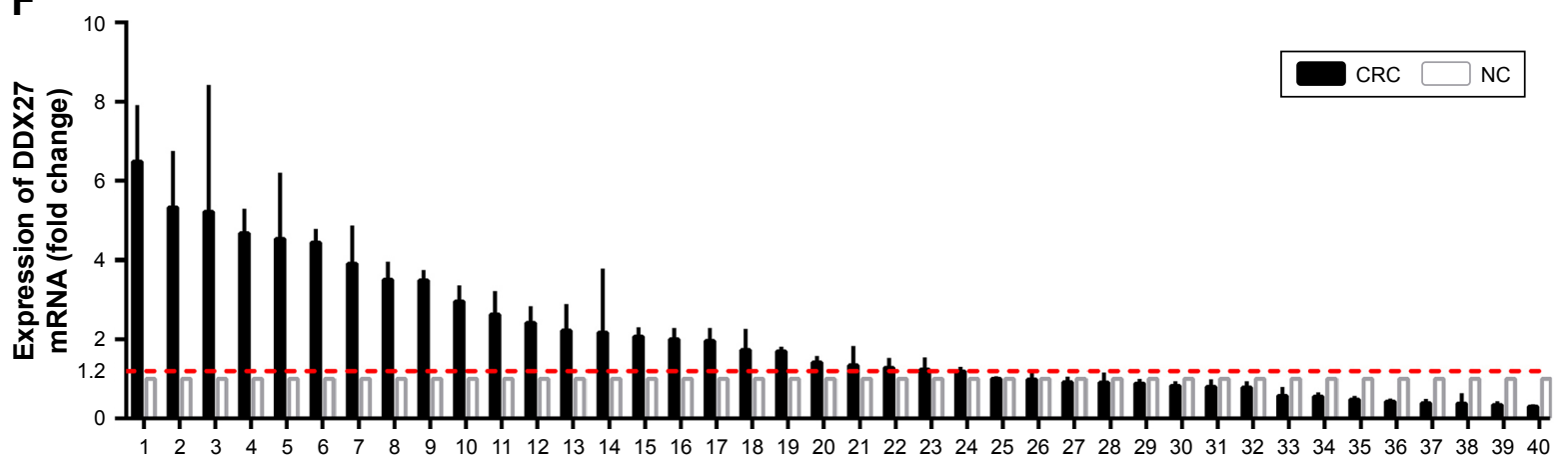

Figure I DDX27 is upregulated in CRC tissues.

Notes: (A) Top ten overexpressed genes in CRC from COSMIC dataset. (B) DDX27 expression information for several cancer types from COSMIC dataset. (C) DDX27 expression level data in CRC from GEO dataset. (D) DDX27 expression level data in CRC from TCGA dataset. (E) Representative images of Western blots performed to examine DDX27 expression in 40 paired human CRC and adjacent normal tissues. (F) qRT-PCR was performed to examine DDX27 expression in 40 paired human CRC and adjacent normal tissues. The expression of DDX27 was normalized to GAPDH. Data were analyzed using a $2^{-\Delta \Lambda} \mathrm{Ct}$ approach. All data are shown as the mean \pm SD for three independent experiments $(* * * * P<0.000 \mathrm{I})$.

Abbreviations: CNV, copy number variations; COSMIC, Catalog Of Somatic Mutations In Cancer; CRC, colorectal cancer; GEO, Gene Expression Omnibus; N, normal tissue; NC, negative control; qRT-PCR, quantitative reverse transcriptase-PCR; T, tumor. 
for which CCK-8 as well as colony-formation assays were performed. The results showed that the DDX27 knockdown remarkably inhibited cell proliferation (Figure 2A-D). Further, flow cytometry was conducted to analyze the effect of DDX27 on cell cycle in CRC cells. The results indicated that DDX27 knockdown retarded G1/S transition, namely, the number of cells in the G1 phase was increased, but the numbers of cells in the $\mathrm{S}$ and G2/M phases were reduced,
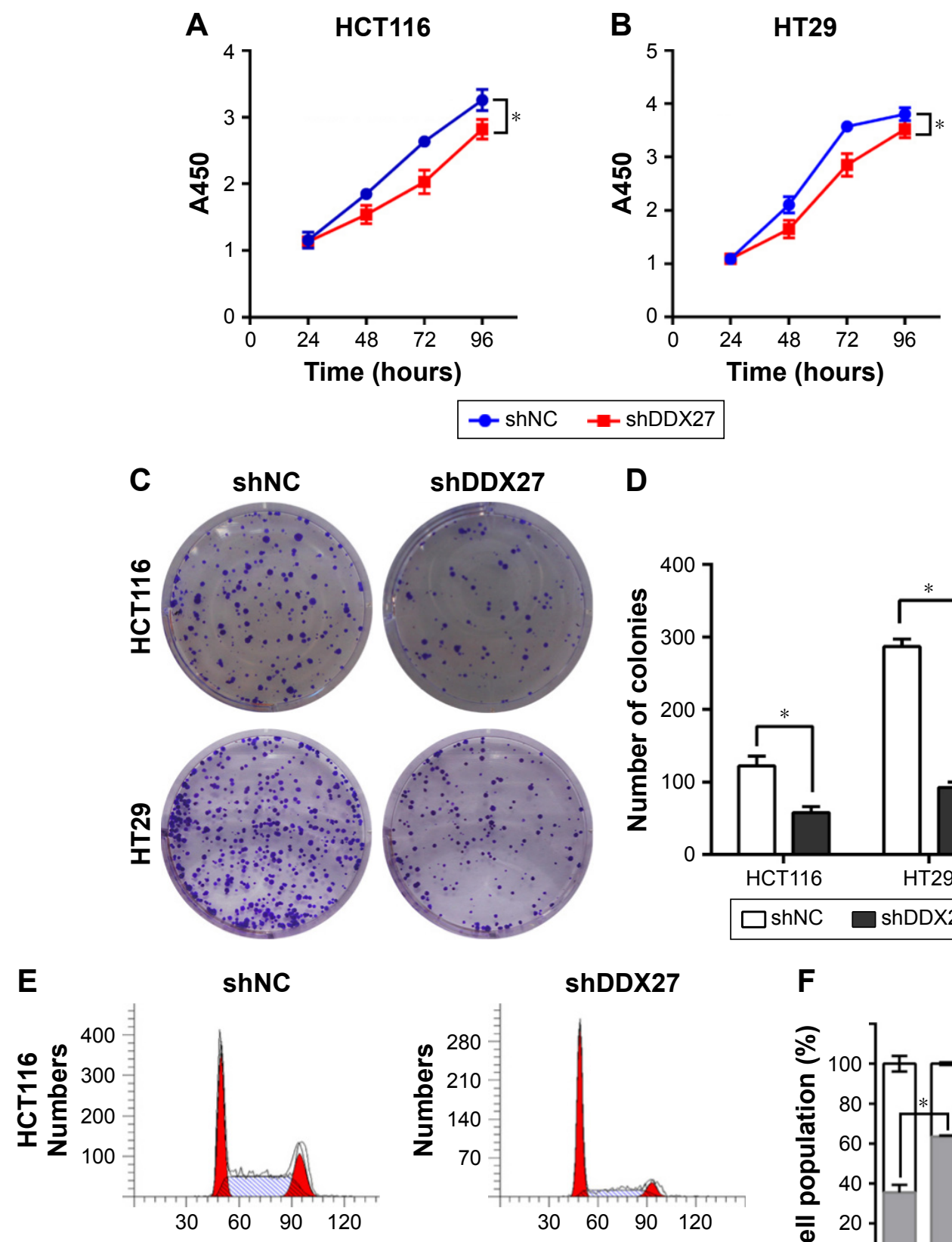

Propidium iodide

E

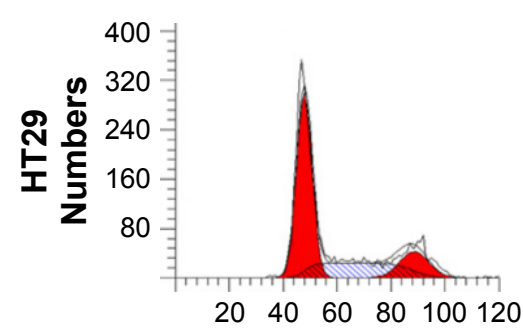

Propidium iodide

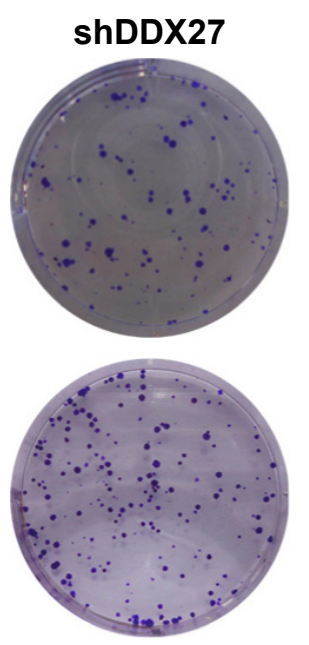

shDDX27

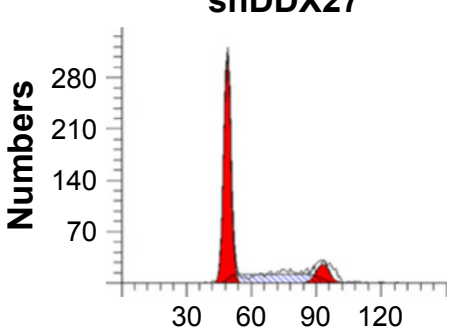

Propidium iodide

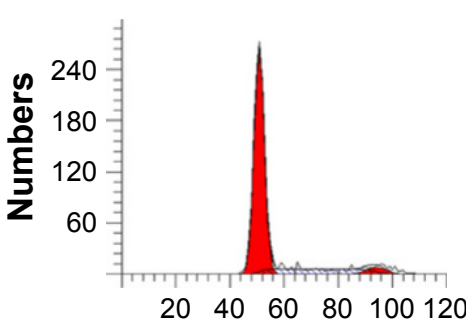

Propidium iodide

Figure 2 (Continued) 

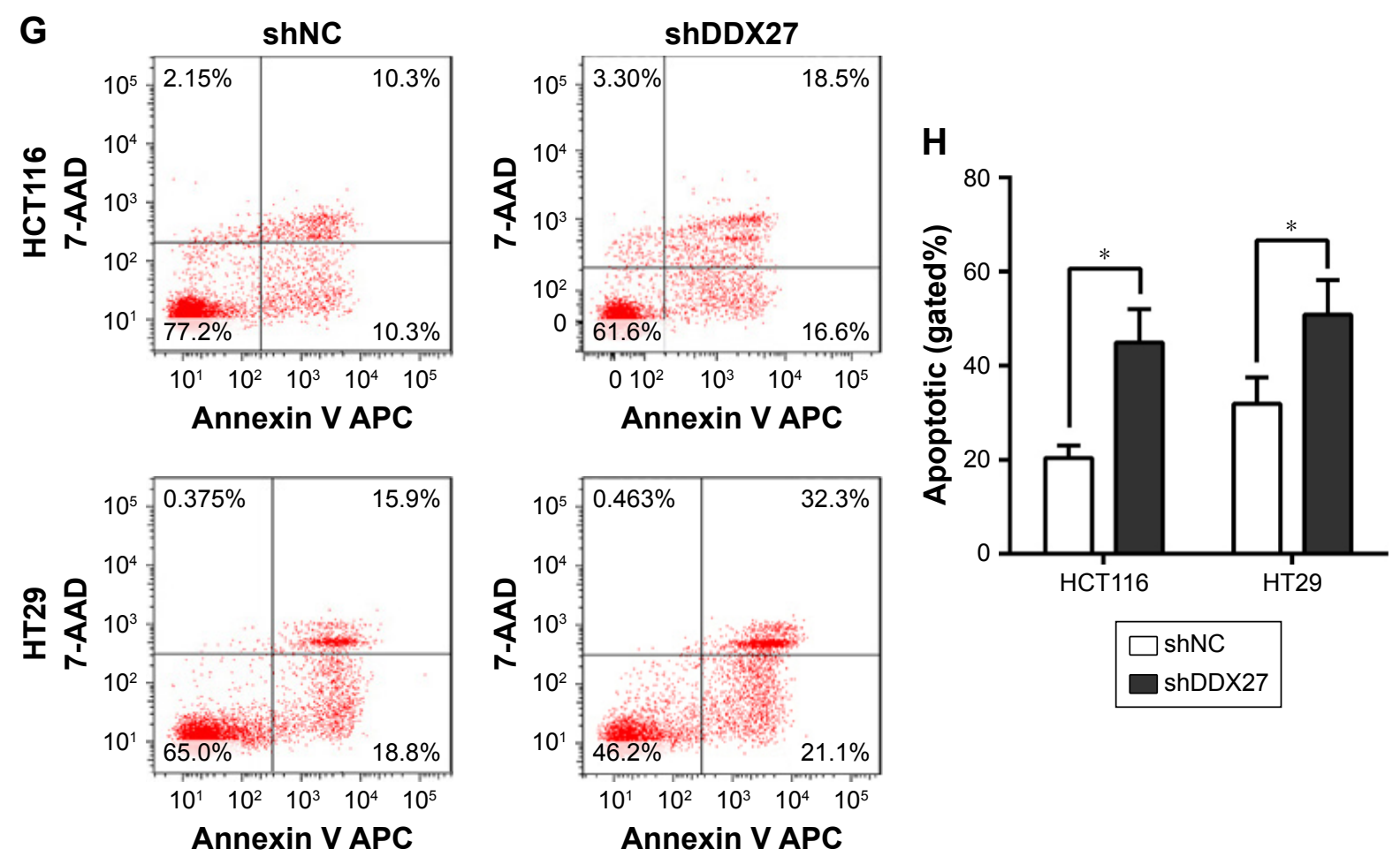

Figure 2 Downregulation of DDX27 inhibits CRC cell proliferation and promotes sensitivity to 5-FU.

Notes: (A, B) CCK-8 assay indicates that knockdown of DDX27 represses CRC cell proliferation. (C, D) Colony-formation assay indicates that knockdown of DDX27I represses CRC cell proliferation. (E, F) Cell cycle analysis reveals that DDX27 influences the proliferation of HT29 and HCTI I6 cells by regulating the cell cycle. (G, H) Cell apoptosis analysis reveals that DDX27 influences the 5-FU sensitivity of HCTII6 and HT29 cells. $* P<0.05$, the means \pm SD are shown.

Abbreviations: 5-FU, 5-fluorouracil; 7-AAD, 7-aminoactinomycin D; APC, allophycocyanin; CCK-8, Cell Counting Kit-8; CRC, colorectal cancer; NC, negative control.

in comparison to the sh-NC group (Figure $2 \mathrm{E}$ and F). These results indicate that DDX27 knockdown repressed cell growth in HT29 and HCT116 cells. We also explored the influence of DDX27 on CRC cells' sensitivity to 5-FU in vitro. DDX27 knockdown led to $\sim 2.2$-fold and $\sim 1$.6-fold increases in apoptotic cell death of HCT116 and HT29 cells, respectively, treated with 5-FU, as analyzed by flow cytometric analysis (Figure $2 \mathrm{G}$ and $\mathrm{H}$ ).

\section{Downregulation of DDX27 weakens} the self-renewal ability and enhances the differentiation potential of colonospheres We checked the GSE14773 profile and found that the DDX27 expression level in HT29 first spheres was higher than in HT29 parental cells, which implied that DDX27 may be associated with the sphere-formation ability (Figure 3A). Therefore, we wanted to explore whether DDX27 plays a tumor-promoter role by regulating the stem cell-like activity of human CRC cells in vitro. Compared with shNC, the expression levels of various stemness genes in shDDX27 were significantly lower, whereas the levels of differentiation markers TFF3 and MUC2 were higher in HT29 (Figure 3B). Then, a sphere-formation assay showed that the silencing of DDX27 could weaken the sphere-formation potential of HT29 cells, both in 500- and 1,000-seeded cell groups. HT29 shDDX27 cells formed fewer and smaller stem-like colonospheres, which means that HT29 shDDX27 cells have weaker self-renewal ability compared with HT29 shNC cells (Figure 3C and D). To determine the differentiation potential of these colonospheres, they were cultivated without EGF and FGF- $\beta$ and in the presence of $2 \%$ FBS. The differentiation process was faster in HT29 shDDX27 than in HT29 shNC. As time went on, floating undifferentiated cells attached to the plastic, gradually migrating from colonospheres and differentiating into large and adherent cells (Figure 3E).

\section{Downregulation of DDX27 decreases the tumor-initiating ability of CRC cells in vivo}

A subcutaneous tumor-formation assay in immunodeficient mice is one approach typically used to evaluate CSC phenotype and activity in published studies. ${ }^{19}$ Thus, we determined whether DDX27 plays a tumor-promoter role by regulating the stem cell-like activity of human CRC cells in vivo. Our subcutaneous tumor-formation assay showed that stemlike spherical cells were more efficient in producing bulky 
A

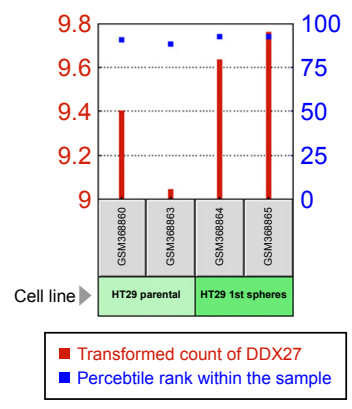

E
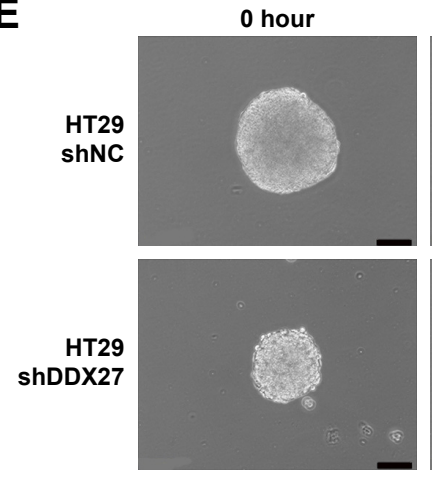

B

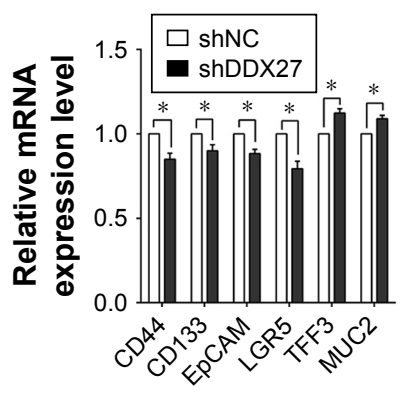

C

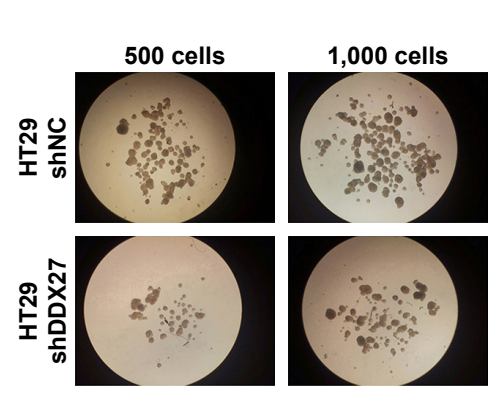

D

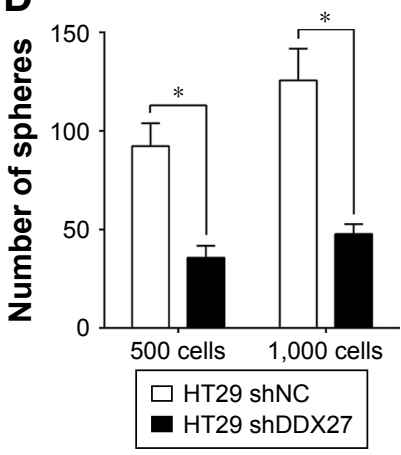

Figure 3 Downregulation of DDX27 weakens the self-renewal ability and enhances the differentiation potential of colonospheres.

Notes: (A) DDX27 expression level in HT29 first spheres and HT29 parental cells from the GDS45II/2I5693_X_at profile. (B) qRT-PCR validation of stemness and differentiation genes' expression in HT29 shNC and shDDX27 cells. The mean expression levels of the target genes were normalized to GAPDH. (C, D) Spheres formed per 500 or 1,000 seeded cells as an index of cell renewal ability ( $4 \times$ microscope magnification). (E) Representative cell images upon differentiation induced by $2 \%$ FBS medium of colonospheres. Bar $=100 \mu \mathrm{m}$. $* P<0.01$, means $\pm S D$ are shown.

Abbreviations: NC, negative control; qRT-PCR, quantitative reverse transcriptase-PCR.

tumors in mice, but shDDX27 could partially reverse this effect. In detail, the tumor-initiating ability of HT29 shNC colonospheres, HT29 shDDX27 colonospheres, HT29 shNC parental, and HT29 shDDX27 parental groups were 5/5, 4/5, $3 / 5$, and $1 / 5$, respectively (Figure $4 \mathrm{~A}$ and B). Additionally, the more efficient the tumor-initiating ability, the bigger is the average tumor volume (Figure 4C).

\section{Discussion}

In this study, we evaluated the role of DDX27 in modulating CSC phenotype at the level of proliferation, sensitivity to 5-FU, sphere formation, CSC marker expression, and tumorinitiating potential of CRC cells. We found that the knockdown of DDX27 increased the sensitivity to 5-FU and decreased the proliferation, sphere formation, gene expression of known CSC markers (CD44, CD133, EpCAM, LGR5), as well as the tumor-initiating potential of CRC cells. This outcome means that high DDX27 expression decreased cell sensitivity to 5-FU and stabilized the phenotype of self-renewing of CRC cells, slowed down their differentiation toward non-stem-like states, and resulted in more efficient tumor initiation.
Genetic abnormalities are a hallmark of cancer. ${ }^{20}$ Our analysis of independent datasets indicates that DDX27 was upregulated in CRC samples compared with adjacent normal tissue (overexpression percentage reached $59.84 \%$ in COSMIC dataset), which indicated that DDX27 may play a promoter role in CRC. The quantitative reverse transcriptasePCR results of our 40 paired clinical CRC samples (overexpression reached $57.50 \%$ ) were consistent with the datasets and data of another study on DDX27 in CRC. ${ }^{21}$ This information indicates that DDX27 may be a potential diagnostic marker. However, the number of samples analyzed was small, which was the major limitation of our study.

Drug resistance is the main cause of cancer treatment failure. ${ }^{22}$ Touil et al found that 5-FU-resistant subpopulation expresses higher levels of CSC markers, including CD44 and CD133, in CRC cell lines. ${ }^{11}$ Our study showed that DDX27 knockdown could reduce the gene expression of known CSC markers (CD44, CD133, EpCAM, LGR5) and enhance the sensitivity to 5-FU in both HCT116 and HT29 CRC cell lines. Our research supports the notion that CSCs' features are associated with drug resistance in CRC. 
A

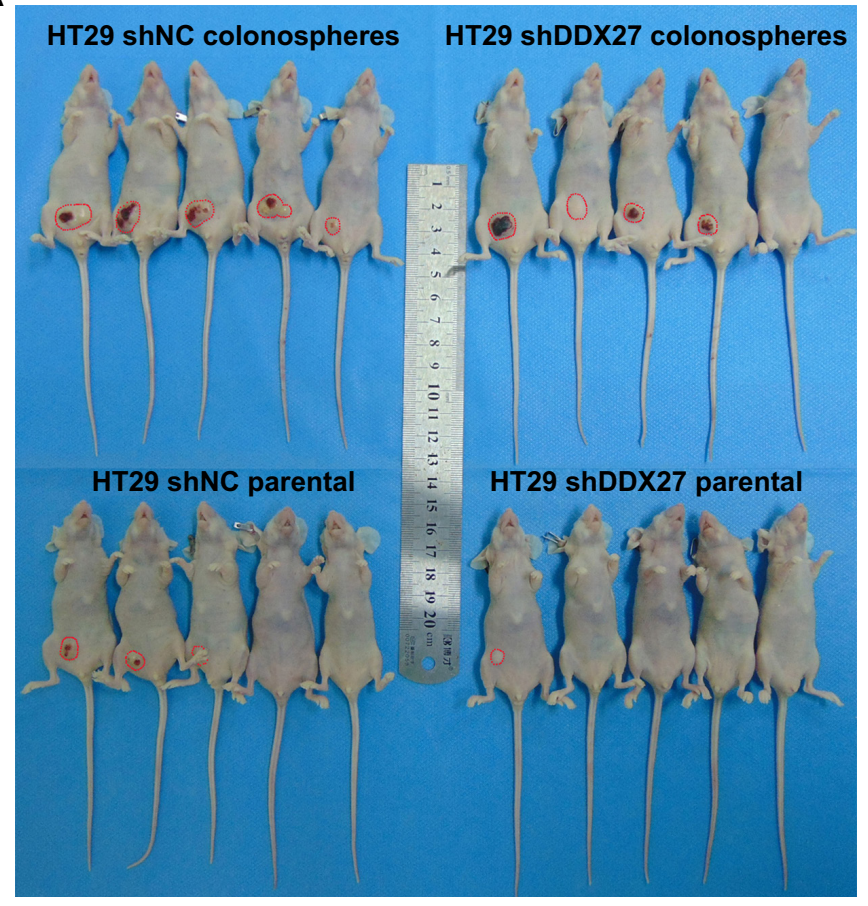

B
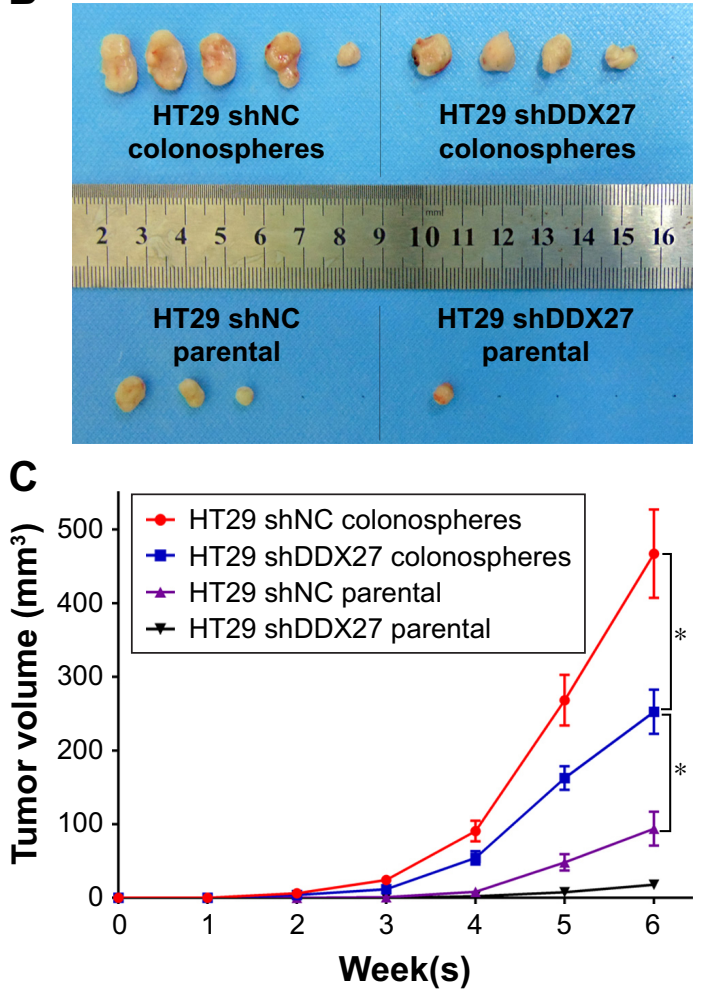

Figure 4 Downregulation of DDX27 decreases the tumor-initiating ability of colorectal cancer cells in vivo.

Notes: (A) Tumor-initiating potential associated with HT29 shNC or shDDX27 colonospheres and parental cells in Balb/c nude mice. (B) The individual tumor body surgical resection from $(\mathbf{A})$. (C) Increased tumor volume in mice at a dose of $1 \times 10^{5}$ cells using $\mathrm{HT} 29$ shNC or shDDX27 colonospheres and parental cells. $* P<0.05$, means $\pm \mathrm{SD}$ are shown.

Abbreviation: NC, negative control.

Sphere-formation assay and subcutaneous tumorformation assay in immunodeficient mice are two different approaches that have typically been used to evaluate CSC phenotype and activity in published studies. ${ }^{19}$ These two approaches were used, and we determined that the knockdown of DDX27 expression in CRC cells reduced the self-renewing capacity of CSCs both in vitro and in vivo. Importantly, the silencing of DDX27 can weaken the tumorinitiating potential of CRC cells in vivo. The overpopulation of CSCs is a driving factor that contributes to colon tumorigenesis, ${ }^{23}$ and knockdown of DDX27 can reduce the gene expression of known SC markers (CD44, CD133, EpCAM, LGR5), which means that it can reduce the population of CSCs. Our findings show a promoter effect of DDX27 on CSC-like cell self-renewal, and self-renewal is closely coupled with proliferation. ${ }^{24}$ Interestingly, DDX17, from the same family as DDX27, was reported to promote stem-like features of estrogen receptor-positive breast cancer. ${ }^{25}$

\section{Conclusion}

Our findings indicate that high expression of DDX27 in CRC cell lines promotes CSC self-renewal and hampers their transition toward more differentiated phenotypes, thus contributing to CSC overpopulation that drives CRC development. This provides a possibility for the potential development of targeted drugs as prospective tools to decrease the self-renewal potential of colorectal CSCs. Altogether, our findings indicate that DDX27 can promote CRC tumorigenesis at least partially by enhancing stem-like features of CRC cells, while the underlying mechanisms need to be further explored.

\section{Acknowledgments}

The present study was supported by the The New Xiangya Talent Projects of the Third Xiangya Hospital of Central South University (No. JY201611).

\section{Disclosure}

The authors report no conflicts of interest in this work.

\section{References}

1. Torre LA, Bray F, Siegel RL, Ferlay J, Lortet-Tieulent J, Jemal A. Global cancer statistics, 2012. CA Cancer J Clin. 2015;65(2):87-108.

2. Chen W, Zheng R, Baade PD, et al. Cancer statistics in China, 2015. CA Cancer J Clin. 2016;66(2):115-132. 
3. Kreso A, O'Brien CA, van Galen $\mathrm{P}$, et al. Variable clonal repopulation dynamics influence chemotherapy response in colorectal cancer. Science. 2013;339(6119):543-548.

4. Siegel R, Desantis C, Jemal A. Colorectal cancer statistics, 2014. CA Cancer J Clin. 2014;64(2):104-117.

5. Clarke MF, Dick JE, Dirks PB, et al. Cancer stem cells - perspectives on current status and future directions: AACR workshop on cancer stem cells. Cancer Res. 2006;66(19):9339-9344.

6. Ajani JA, Song S, Hochster HS, Steinberg IB. Cancer stem cells: the promise and the potential. Semin Oncol. 2015;42(Suppl 1):S3-S17.

7. Clarke MF. Self-renewal and solid-tumor stem cells. Biol Blood Marrow Transplant. 2005;11(2):14-16

8. Vermeulen L, Morrissey E, van der Heijden M, et al. Defining stem cell dynamics in models of intestinal tumor initiation. Science. 2013 342(6161):995-998.

9. Biddle A, Liang X, Gammon L, et al. Cancer stem cells in squamous cell carcinoma switch between two distinct phenotypes that are preferentially migratory or proliferative. Cancer Res. 2011;71(15):5317-5326.

10. Malanchi I, Santamaria-Martínez A, Susanto E, et al. Interactions between cancer stem cells and their niche govern metastatic colonization. Nature. 2012;481(7379):85-89.

11. Touil Y, Igoudjil W, Corvaisier M, et al. Colon cancer cells escape 5FU chemotherapy-induced cell death by entering stemness and quiescence associated with the c-Yes/YAP axis. Clin Cancer Res. 2014;20(4):837-846.

12. Li Y, Rogoff HA, Keates S, et al. Suppression of cancer relapse and metastasis by inhibiting cancer stemness. Proc Natl Acad Sci USA. 2015; 112(6):1839-1844.

13. Fairman-Williams ME, Guenther UP, Jankowsky E. SF1 and SF2 helicases: family matters. Curr Opin Struct Biol. 2010;20(3):313-324.

14. Linder P, Lasko PF, Ashburner M, et al. Birth of the D-E-A-D box Nature. 1989;337(6203):121-122.

15. Linder P, Jankowsky E. From unwinding to clamping - the DEAD box RNA helicase family. Nat Rev Mol Cell Biol. 2011;12(8):505-516.
16. Zhang Y, Baysac KC, Yee L-F, Saporita AJ, Weber JD. Elevated DDX21 regulates c-Jun activity and rRNA processing in human breast cancers. Breast Cancer Res. 2014;16(5):449.

17. Fukawa T, Ono M, Matsuo T, et al. DDX31 regulates the p53-HDM2 pathway and rRNA gene transcription through its interaction with NPM1 in renal cell carcinomas. Cancer Res. 2012;72(22):5867-5877.

18. Tsukamoto Y, Fumoto S, Noguchi T, et al. Expression of DDX27 contributes to colony-forming ability of gastric cancer cells and correlates with poor prognosis in gastric cancer. Am J Cancer Res. 2015;5(10): 2998-3014

19. Takaishi S, Okumura T, Wang TC. Gastric cancer stem cells. J Clin Oncol. 2008;26(17):2876-2882.

20. Bochtler T, Fröhling S, Krämer A. Role of chromosomal aberrations in clonal diversity and progression of acute myeloid leukemia. Leukemia. 2015;29(6):1243-1252.

21. Tang J, Chen H, Wong CC, et al. DEAD-box helicase 27 promotes colorectal cancer growth and metastasis and predicts poor survival in CRC patients. Oncogene. 2018;37(22):3006-3021.

22. Colak S, Medema JP. Cancer stem cells - important players in tumor therapy resistance. FEBS J. 2014;281(21):4779-4791.

23. Boman BM, Fields JZ, Cavanaugh KL, Guetter A, Runquist OA. How dysregulated colonic crypt dynamics cause stem cell overpopulation and initiate colon cancer. Cancer Res. 2008;68(9):3304-3313.

24. Bhatlekar S, Viswanathan V, Fields JZ, Boman BM. Overexpression of HOXA4 and HOXA9 genes promotes self-renewal and contributes to colon cancer stem cell overpopulation. J Cell Physiol. 2018;233(2): 727-735.

25. Alqahtani H, Gopal K, Gupta N, et al. DDX17 (P72), a Sox2 binding partner, promotes stem-like features conferred by Sox 2 in a small cell population in estrogen receptor-positive breast cancer. Cell Signal. $2016 ; 28(2): 42-50$
OncoTargets and Therapy

\section{Publish your work in this journal}

OncoTargets and Therapy is an international, peer-reviewed, open access journal focusing on the pathological basis of all cancers, potential targets for therapy and treatment protocols employed to improve the management of cancer patients. The journal also focuses on the impact of management programs and new therapeutic agents and protocols on

\section{Dovepress}

patient perspectives such as quality of life, adherence and satisfaction. The manuscript management system is completely online and includes a very quick and fair peer-review system, which is all easy to use. Visit http://www.dovepress.com/testimonials.php to read real quotes from published authors. 